\title{
A difícil adesão dos pacientes hipertensos ao tratamento: Revisão de literatura
}

\author{
The difficult adherence of hypertensive patients to treatment: \\ A literature review
}

Silas Santos Carvalho ${ }^{1}$ Bruno Rodrigues de Oliveira ${ }^{1}$ 'Universidade Estadual de Feira de Santana-BA (UEFS). Feira de Santana/BA - Brasil
Resumo Objetivo: Identificar, com base na literatura, os fatores causais que levam os pacientes hipertensos à não adesão ao tratamento.

Métodos: Trata-se de um estudo bibliográfico com caráter exploratório e abordagem qualitativa. O material selecionado para a análise foi constituído por dez artigos publicados entre os anos de 2010 a 2017, disponíveis on-line nas bases de dados SciELO e LILACS. Resultados: Entre as questões que dificultam a adesão ao tratamento do hipertenso, se destacam as relacionadas especialmente aos efeitos das drogas e ao custo da medicação. Quanto ao tratamento não farmacológico, observa-se, com frequência, a falta de constância e persistência do paciente.

Conclusão: É imprescindível identificar as dificuldades relacionadas ao tratamento da hipertensão com vistas a uma melhor adesão dos pacientes hipertensos, bem como, dos que possuem doenças crônicas associadas, reforçando a necessidade de se informar e discutir as condições observadas pelos membros da equipe de saúde.

Palavras-chave: Hipertensão. Tratamento farmacológico. EnFERMAGEM. FATORES DE RISCO.

Abstract Objective: To identify, based on the literature, the causal factors that lead hypertensive patients to non-adherence to treatment.

Methods: This is an exploratory bibliographical study with a qualitative approach. The material selected for analysis consisted of 10 articles published between the years 2010 to 2017, available online in the SciELO and LILACS databases.

Results: Among the issues that make it difficult to comply with the treatment of hypertensive patients, they stand out as products especially related to the effects of drugs and the acquisition of medication. As for the non-pharmacological treatment, the patient's lack of constancy and persistence is observed.

Conclusion: It is essential to identify the difficulties related to the treatment of hypertension with a view to a better adherence of hypertensive patients, as well as the associated chronic diseases, reinforcing the need to inform and discuss as conditions observed by members of the health team.

Key-WORds: Hypertension. Drug therapy. Nursing. Risk factors. 


\section{INTRODUÇÃO}

A Hipertensão Arterial Sistêmica (HAS), popularmente conhecida como pressão alta, é uma das patologias mais frequentes no mundo. Um em cada cinco brasileiros possui a doença, segundo a Organização Mundial de Saúde (OMS). Sabe-se que em qualquer idade o indivíduo pode ser acometido por essa patologia, porém acima dos 50 anos de idade a frequência é mais alta. Essa doença pode aparecer gradativa e silenciosamente, sem apresentar sintomas. ${ }^{1}$

A HAS é uma condição clínica multifatorial que se distingue por níveis elevados e mantidos de pressão arterial (PA). Associa-se, constantemente, a alterações funcionais e/ou estruturais dos órgãos-alvo (coração, encéfalo, rins e vasos sanguíneos) e a alterações metabólicas, com consequente aumento do risco de eventos cardiovasculares fatais e não fatais. ${ }^{2}$

A HAS é uma síndrome silenciosa, considerada um dos maiores problemas na área de saúde pública, seja pela elevada prevalência, cerca de $20 \%$ da população adulta, seja pela acentuada parcela de hipertensos não diagnosticados ou não tratados de forma adequada, ou ainda pelo alto índice de abandono ao tratamento. ${ }^{3}$

Apesar do conhecimento da eficácia e da efetividade de várias medidas de controle preventivas disponíveis, farmacológicas ou não, a hipertensão continuará, por muito tempo, representando um dos maiores desafios em saúde, assim como um dos maiores prejuízos para o portador de HAS e para a sociedade. ${ }^{4}$

Apesar de possuir várias estratégias para estimar a adesão ao tratamento da hi- pertensão, não há consenso sobre um padrão ouro. Os métodos podem ser diretos, como a dosagem do princípio ativo/metabólito da droga ou indiretos, como a contagem de comprimidos e relato do usuário. Alguns autores desenvolveram questionários genéricos para mensurar a adesão ao tratamento de doenças crônicas, também utilizados em pessoas com hipertensão. ${ }^{5}$

Na HAS, o tratamento não farmacológico consiste em estratégias que almejam mudanças no estilo de vida, podendo haver, com isso, diminuição ou até mesmo dispensa dos medicamentos. $O$ tratamento não medicamentoso tem como principal objetivo diminuir a morbidade e a mortalidade cardiovasculares, por meio de modificações do estilo de vida que favoreçam a redução da

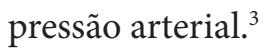

Com base nas considerações já citadas, questiona-se: "Por que muitos pacientes hipertensos não aderem ao tratamento?" A relevância dessa revisão consiste na identificação das causas da não adesão dos pacientes com HAS ao tratamento, visando contribuir para a adoção de estratégias que auxiliem o indivíduo a adotar um comportamento mais efetivo no que diz respeito à doença. Faz-se necessário a existência de um relacionamento com movimento bidirecional entre os atores envolvidos, minimizando possíveis complicações, comorbidade e/ou mortalidade pela falta de compromisso do hipertenso com o tratamento. É necessário nortear os profissionais de saúde no trabalho de motivação do hipertenso que precisa aderir ao tratamento da HAS e também de prevenção a riscos de saúde, o que pode gerar onerosidade aos serviços de saúde e fortificar a prática da 
enfermagem, como coparticipante ativa na assistência à saúde.

Diante disso, o objetivo deste trabalho foi identificar, com base na literatura, os fatores causais que levam os pacientes portadores de hipertensão arterial à não adesão ao tratamento.

\section{Metodologia}

Este artigo se caracteriza como uma pesquisa bibliográfica de natureza descritivo-exploratória, acerca da difícil adesão dos pacientes hipertensos ao tratamento. Nessa perspectiva, foram identificadas as dificuldades de não adesão ao tratamento da hipertensão, bem como, as suas consequências, objetivando apresentar soluções viáveis e efetivas para a redução do problema exibido. A partir dessas delimitações, é perceptível o viés exploratório desta pesquisa.

O material selecionado para a análise foi constituído por dez artigos publicados entre os anos de 2010 e 2017, disponíveis on-line na íntegra, na base de dados Scientific Eletronic Library Online (SCIELO) e Literatura Latino-Americana e do Caribe em Ciências da Saúde (LILACS) e com idioma em português, espanhol e inglês. As palavras-chave utilizadas para busca foram: Hipertensão; Tratamento farmacológico; Enfermagem; Fatores de risco.

Foram excluídos os estudos de resumos, monografias, dissertações, teses, revisões sistemáticas, artigos de opinião e revisões de literatura. As buscas ocorreram a partir do emprego dos seguintes descritores: "enfermagem" AND "hipertensão arterial” AND "tratamento". Procedeu-se à leitura dos títulos das publicações, identificação das que abordavam e as que se distanciavam da temática e, após a compilação dos dados, o referencial teórico foi organizado e construído.

Após a análise dos resumos, todos os artigos selecionados foram obtidos na íntegra. Seguiu-se para a organização, e tabulação dos dados, um roteiro de coleta de dados contendo: autor(es), ano de publicação, objetivo do estudo, metodologia, principais resultados e conclusões encontrados em relação à adesão de pacientes hipertensos. Posteriormente, os estudos foram examinados de acordo com as três categorias para a discussão no presente trabalho: "A família como sujeito/objeto do cuidado de enfermagem", essa categoria engloba as vivências da família relacionadas à sua participação no cuidado ao familiar com HAS ou às consequências geradas pela doença; "Reflexos do acolhimento no cuidado dos profissionais de saúde na atenção ao paciente com hipertensão" e "Importância da adesão do tratamento na qualidade dos cuidados da hipertensão arterial”.

Destaca-se que as informações foram extraídas por meio de um roteiro validado para a organização sistemática e, posteriormente, a classificação em categorias temáticas. ${ }^{6}$ Por ser um artigo de revisão de literatura, não foi necessária a aprovação no Comitê de Ética em Pesquisa. Todavia, foram respeitados os aspectos éticos na pesquisa, bem como, os direitos autorais.

\section{Resultados E Discussão}

A busca nas bases de dados, por meio dos descritores selecionados, resultou em 4.872 títulos, 112 na base SciELO e 4.760 na 
LILACS. A primeira seleção eliminou 3.133 títulos. Em seguida, procedeu-se à análise do conteúdo dos resumos dos 1.739 estudos restantes, dos quais 1.729 foram eliminados por não atenderem aos critérios previamente estabelecidos. Os dez artigos restantes foram lidos na íntegra e compuseram esta revisão. No Quadro 1, encontram-se sumarizados os artigos identificados nesta revisão. Todos os estudos analisados foram desenvolvidos no Brasil, no entanto, dois (20\%) artigos foram publicados em inglês. As categorias foram desenvolvidas com base no processo de assistência dos profissionais de enfermagem como cuidadores de pacientes hipertensos.

Os artigos foram identificados com o número sequencial correspondente precedido pela letra $\mathrm{A}$.

Quadro 1 - Artigos selecionados nas bases de dados para a análise.

\begin{tabular}{|c|c|c|c|c|}
\hline $\begin{array}{l}\text { Código do estudo/ } \\
\text { Autor/Ano }\end{array}$ & Objetivo & Metodologia & Principais resultados & Conclusões \\
\hline $\begin{array}{l}\text { Al. } \\
\text { Bezerra, Lopes, } \\
\text { Barros, } 2014^{8}\end{array}$ & $\begin{array}{l}\text { Identificar a adesão à } \\
\text { terapêutica medicamentosa } \\
\text { em hipertensos, bem como, } \\
\text { os fatores, diretamente } \\
\text { relacionados aos pacientes e que } \\
\text { estão associados a essa adesão. }\end{array}$ & $\begin{array}{l}\text { Estudo descritivo, } \\
\text { corte transversal com } \\
\text { uma amostra de } 77 \\
\text { pacientes, por meio } \\
\text { de um questionário } \\
\text { semiestruturado. }\end{array}$ & $\begin{array}{l}\text { Adesão de } 87 \% \text { dos hipertensos. Os } \\
\text { fatores diretamente relacionados ao } \\
\text { paciente, que se associavam à adesão } \\
\text { foram: a procedência, a autoavaliação } \\
\text { do paciente quanto aos níveis } \\
\text { pressóricos; a vontade de abandono } \\
\text { do tratamento e a aceitação do } \\
\text { paciente quanto à doença. }\end{array}$ & $\begin{array}{l}\text { A maioria dos pacientes hipertensos } \\
\text { avaliados era aderente ao tratamento e } \\
\text { os fatores, diretamente relacionados ao } \\
\text { paciente, que interferiram na adesão } \\
\text { foram: a procedência, a avaliação } \\
\text { do controle dos níveis pressóricos, } \\
\text { sentimento para abandono do } \\
\text { tratamento e aceitação da doença. }\end{array}$ \\
\hline $\begin{array}{l}\text { A2. } \\
\text { Figueiredo, Asakura, } \\
2010^{9}\end{array}$ & $\begin{array}{l}\text { Caracterizar pacientes } \\
\text { hipertensos e conhecer suas } \\
\text { principais dificuldades para } \\
\text { aderir ao tratamento proposto } \\
\text { pela equipe de saúde. }\end{array}$ & $\begin{array}{l}\text { Estudo descritivo, corte } \\
\text { transversal com pacientes } \\
\text { aleatoriamente } \\
\text { selecionados e consulta } \\
\text { de prontuários } \\
\text { daqueles que estavam em } \\
\text { seguimento ambulatorial. }\end{array}$ & $\begin{array}{l}\text { Entre os } 54 \text { pacientes hipertensos } \\
66,7 \% \text { eram do sexo feminino, } \\
\text { adultos com idade entre } 30 \text { e } 90 \\
\text { anos. As médias da pressão arterial } \\
\text { (mmHg) sistólica e diastólica } \\
\text { foram 138 e 83, respectivamente, } \\
\text { mas apenas metade dos pacientes } \\
\text { apresentava pressão arterial } \\
\text { controlada. Observou-se associação } \\
\text { entre o conhecimento sobre a doença } \\
\text { e o seguimento das orientações } \\
\text { para o tratamento da hipertensão. } \\
\text { A principal dificuldade relatada } \\
\text { foi seguir dieta hipossódica. } \\
\text { Comparando-se as orientações } \\
\text { prescritas com aquelas que os } \\
\text { pacientes referiram ter recebido, } \\
\text { houve concordância em } 37 \text { casos. }\end{array}$ & $\begin{array}{l}\text { A comunicação entre os membros da } \\
\text { equipe de sauude e os pacientes deve ser } \\
\text { melhorada, e as pessoas envolvidas no } \\
\text { tratamento da hipertensão precisam } \\
\text { discutir as dificuldades encontradas e } \\
\text { buscar formas de resolvê-las. }\end{array}$ \\
\hline $\begin{array}{l}\text { A3. } \\
\text { Duarte et al., } 2010^{10}\end{array}$ & $\begin{array}{l}\text { Identificar as razões expostas } \\
\text { por portadores de HAS para } \\
0 \text { abandono do seguimento } \\
\text { médico em serviço de atenção } \\
\text { primária à saúde e as estratégias } \\
\text { que empregam para o controle } \\
\text { da hipertensão arterial. }\end{array}$ & $\begin{array}{l}\text { Estudo de coorte } \\
\text { com } 192 \text { usuários } \\
\text { diagnosticados } \\
\text { com hipertensão, } \\
\text { acompanhados num } \\
\text { período compreendido } \\
\text { de três anos e meio. }\end{array}$ & $\begin{array}{l}\text { Os motivos relatados para o } \\
\text { abandono do seguimento mostraram } \\
\text { predomínio de razões ligadas ao } \\
\text { próprio serviço de saúde e a relação } \\
\text { médico-paciente, tratamento em } \\
\text { outro serviço de saúde, ausência } \\
\text { de sintomas, a melhora e/ou a } \\
\text { normalização da pressão arterial e o } \\
\text { consumo de álcool. }\end{array}$ & $\begin{array}{l}\text { O estudo permitiu verificar a riqueza e a } \\
\text { diversidade de problemas envolvidos no } \\
\text { cuidado requerido. }\end{array}$ \\
\hline $\begin{array}{l}\text { A4. } \\
\text { Ribeiro et al., } 2015^{4}\end{array}$ & $\begin{array}{l}\text { Avaliar a prevalência e os } \\
\text { fatores associados à adesão ao } \\
\text { tratamento medicamentoso por } \\
\text { pacientes hipertensos. }\end{array}$ & $\begin{array}{l}\text { Estudo transversal e } \\
\text { descritivo. Utilizou-se } \\
\text { questionário na coleta } \\
\text { de dados. }\end{array}$ & $\begin{array}{l}\text { Evidenciou-se prevalência de } 72,8 \% \\
\text { de adesão ao tratamento; diferenças } \\
\text { significativas na adesão segundo } \\
\text { situação marital de união estável } \\
\text { ( } \mathrm{p}=0,043) \text {, controle da PA por meio } \\
\text { de tratamento medicamentoso } \\
\text { combinado com mudanças no } \\
\text { estilo de vida ( } \mathrm{p}=0,003) \text { e tempo de } \\
\text { diagnóstico da hipertensão inferior a } \\
10 \text { anos }(\mathrm{p}<0,001) \text {. }\end{array}$ & $\begin{array}{l}\text { A adesão ao tratamento medicamentoso } \\
\text { para a HAS pôde ser considerada } \\
\text { satisfatória na amostra estudada; e } \\
\text { apresentaram melhores níveis de adesão } \\
\text { indivíduos em situação marital de união } \\
\text { estável, com controle da PA por meio } \\
\text { do tratamento medicamentoso aliado } \\
\text { à adoção de hábitos de vida saudáveis e } \\
\text { tempo de diagnóstico da HAS inferior } \\
\text { a } 10 \text { anos. }\end{array}$ \\
\hline
\end{tabular}




\begin{tabular}{|c|c|c|c|c|}
\hline $\begin{array}{l}\text { Código do estudo/ } \\
\text { Autor/Ano }\end{array}$ & Objetivo & Metodologia & Principais resultados & Conclusões \\
\hline $\begin{array}{l}\text { A5. } \\
\text { Almeida, Paz, Silva, } \\
2013^{13}\end{array}$ & $\begin{array}{l}\text { Analisar as representações } \\
\text { dos usuários de serviços } \\
\text { básicos de saúde portadores } \\
\text { de hipertensão arterial sobre } \\
\text { a doença e a maneira como se } \\
\text { cuidam. }\end{array}$ & $\begin{array}{l}\text { Entrevista } \\
\text { semiestruturada e } \\
\text { técnica do discurso do } \\
\text { sujeito coletivo para a } \\
\text { organização dos dados } \\
\text { e interpretação pela } \\
\text { teoria das representações } \\
\text { sociais. }\end{array}$ & $\begin{array}{l}\text { Conhecimento insuficiente dos } \\
\text { usuários sobre a hipertensão } \\
\text { arterial e os meios para o controle e } \\
\text { tratamento da doença. Reconhecem } \\
\text { a hipertensão como uma patologia } \\
\text { grave, que pode trazer grandes } \\
\text { consequências quando não tratada. }\end{array}$ & $\begin{array}{l}\text { As dificuldades estruturais dos serviços } \\
\text { contribuem para desmotivar o usuário } \\
\text { a participar de ações de promoção } \\
\text { em saúde. Tais resultados indicam } \\
\text { a necessidade de intensificação das } \\
\text { ações assistenciais que sensibilizem os } \\
\text { portadores da doença para a busca pelo } \\
\text { controle consciente da hipertensão. }\end{array}$ \\
\hline $\begin{array}{l}\text { A6. } \\
\text { Medeiros et al., } \\
2014^{18}\end{array}$ & $\begin{array}{l}\text { Desenvolver um modelo de } \\
\text { apoio à tomada de decisão } \\
\text { para identificar indivíduos não } \\
\text { aderentes ao tratamento anti- } \\
\text { hipertensivo. }\end{array}$ & $\begin{array}{l}\text { Estudo observacional, } \\
\text { seccional com } 118 \\
\text { usuários hipertensos. } \\
\text { Utilizou-se a técnica } \\
\text { de entrevista com } \\
\text { aplicação de questionário } \\
\text { estruturado. }\end{array}$ & $\begin{array}{l}\text { A variável de decisão foi a adesão } \\
\text { ao tratamento. Oito variáveis entre } \\
\text { os } 15 \text { atributos foram utilizadas } \\
\text { na construção da árvore decisória: } \\
\text { dificuldade em ir ao posto de saúde, } \\
\text { conhecimento sobre HAS, medo do } \\
\text { tratamento, condição afetiva, sexo, } \\
\text { ocupação, opinião sobre o número } \\
\text { de medicamentos utilizados no } \\
\text { tratamento e controle da PA. }\end{array}$ & $\begin{array}{l}\text { O modelo de árvore de decisão proposto } \\
\text { auxilia na identificação de usuários não } \\
\text { aderentes, de modo a contribuir com as } \\
\text { equipes de saúde na abordagem a esses } \\
\text { indivíduos. }\end{array}$ \\
\hline $\begin{array}{l}\text { A7. } \\
\text { Machado et al., } \\
2016^{21}\end{array}$ & $\begin{array}{l}\text { Comparar o efeito de três } \\
\text { estratégias de educação em } \\
\text { saúde e nutrição sobre a } \\
\text { adesão ao tratamento não } \\
\text { farmacológico da HAS, pelos } \\
\text { parâmetros antropométricos, } \\
\text { bioquímicos, clínicos e } \\
\text { dietéticos. }\end{array}$ & $\begin{array}{l}\text { Estudo de intervenção } \\
\text { longitudinal, } \\
\text { comparativo, do tipo } \\
\text { ensaio comunitário, } \\
\text { realizado com } 212 \\
\text { indivíduos hipertensos. }\end{array}$ & $\begin{array}{l}\text { Entre os diferentes grupos, a redução } \\
\text { da glicose mostrou diferença } \\
\text { estatisticamente significante, tendo } \\
\text { o Grupo } 2 \text { apresentado melhor } \\
\text { evolução. Os Grupos } 1 \text { e } 2 \text { tiveram } \\
\text { melhores resultados sobre a adesão } \\
\text { ao tratamento não farmacológico } \\
\text { da HAS. }\end{array}$ & $\begin{array}{l}\text { Na prática dos serviços de saúde, há o } \\
\text { desafio de se promover uma educação } \\
\text { em saúde capaz de intervir sobre a } \\
\text { problemática da adesão ao tratamento. }\end{array}$ \\
\hline $\begin{array}{l}\text { A8. } \\
\text { Ungari, Dal Fabbro, } \\
2010 . .^{23}\end{array}$ & $\begin{array}{l}\text { Estudar a adesão ao tratamento } \\
\text { farmacológico de pacientes } \\
\text { hipertensos seguidos no } \\
\text { Programa de Saúde da Família. }\end{array}$ & $\begin{array}{l}\text { Estudo transversal com } \\
109 \text { pacientes, utilizando- } \\
\text { se o Teste de Morisky e } \\
\text { Green para mensurar } \\
\text { o grau de adesão ao } \\
\text { tratamento. }\end{array}$ & $\begin{array}{l}\text { Os resultados do teste foram } \\
\text { avaliados por dois critérios: os } \\
\text { pacientes foram classificados como } \\
\text { "mais aderentes" se apresentassem } \\
\text { pontuação de } 3 \text { a } 4 \text { (critério 1) ou de } \\
4 \text { (critério 2); pacientes com outras } \\
\text { pontuações foram classificados } \\
\text { como "menos aderentes". Dos } \\
\text { pacientes, } 79,8 \% \text { e } 43,1 \% \text { foram } \\
\text { "mais aderentes" de acordo com os } \\
\text { critérios } 1 \text { e 2, respectivamente. Em } \\
\text { relação às possíveis causas da não } \\
\text { adesão, observaram-se associações } \\
\text { significativas (p<0,05) entre as } \\
\text { variáveis "confiança no médico" ou } \\
\text { "quantidade de medicamentos anti- } \\
\text { hipertensivos que utiliza" e "grau de } \\
\text { adesão", pelo critério } 2 \text {. }\end{array}$ & $\begin{array}{l}\text { Pacientes apresentam graus } \\
\text { satisfatórios de adesão ao tratamento } \\
\text { medicamentoso, a importância da } \\
\text { interação profissional/paciente, a } \\
\text { confiança no médico e a atitude dos } \\
\text { profissionais de saúde em relação ao } \\
\text { usuário. }\end{array}$ \\
\hline $\begin{array}{l}\text { A9. } \\
\text { Santos et al., } 2013 .^{24}\end{array}$ & $\begin{array}{l}\text { Analisar a adesão do usuário } \\
\text { hipertenso ao tratamento } \\
\text { medicamentoso. }\end{array}$ & $\begin{array}{l}\text { Estudo descritivo com } \\
400 \text { hipertensos por } \\
\text { intermédio de entrevista. }\end{array}$ & $\begin{array}{l}\text { A maioria dos entrevistados era } \\
\text { mulher }(67,2 \%) \text {, tinha idade acima } \\
\text { dos } 60 \text { anos }(54,3 \%) \text {, e pardos } \\
\text { (54,7\%). Cerca de } 81,5 \% \text { faziam } \\
\text { uso regular do medicamento com } \\
\text { predomínio dos entrevistados com } \\
\text { mais de dez anos de diagnóstico } \\
\text { (33,5\%), até } 5 \text { anos de tratamento } \\
\text { (31,0\%), e que recebiam a medicação } \\
\text { completa do Sistema Único de Saúde } \\
(39,2 \%) \text {. }\end{array}$ & $\begin{array}{l}\text { O saber sobre os tipos de drogas } \\
\text { anti-hipertensivas e o número } \\
\text { de tomadas diárias predominou } \\
\text { independentemente da regularidade } \\
\text { da medicação, o mesmo fato aconteceu } \\
\text { em relação ao desconhecimento dos } \\
\text { efeitos colaterais, aos desconfortos } \\
\text { causados, e à complexidade do esquema } \\
\text { medicamentoso. A adesão ao tratamento } \\
\text { prevalecia na maioria dos usuários. }\end{array}$ \\
\hline $\begin{array}{l}\text { A10. } \\
\text { Serafim, Jesus, } \\
\text { Pierin, } 2010^{25}\end{array}$ & $\begin{array}{l}\text { Caracterizar hábitos de vida } \\
\text { e conhecimentos sobre eles } \\
\text { associando variáveis estudadas } \\
\text { com o controle da hipertensão } \\
\text { arterial em pessoas atendidas } \\
\text { em ambulatório. }\end{array}$ & $\begin{array}{l}\text { Estudo descritivo } \\
\text { utilizando entrevistas e } \\
\text { mensuração da pressão } \\
\text { arterial, peso, altura e } \\
\text { circunferência abdominal } \\
\text { de } 511 \text { pessoas } \\
\text { hipertensas em controle } \\
\text { ambulatorial. }\end{array}$ & $\begin{array}{l}\text { Encontrou-se o Índice de Massa } \\
\text { Corporal no limite superior da } \\
\text { faixa de sobrepeso }(29,04 \pm 4,35 \mathrm{~kg} / \\
\text { m2); a pressão arterial era maior } \\
\text { que o limite caracterizador da HAS } \\
\text { ( } 151,3 \pm 20,5 / 91,8 \pm 15,5 \mathrm{mmHg}) ; \\
44 \% \text { referiram tabagismo ou ex- } \\
\text { tabagismo; } 59 \% \text { não praticavam } \\
\text { atividade física regular; e } 24 \% \\
\text { referiram ingestão ou interrupção } \\
\text { de bebida alcoólica. Apenas } 22 \% \text { dos } \\
\text { hipertensos estavam controlados. }\end{array}$ & $\begin{array}{l}\text { Os participantes que conheciam que } \\
\text { o tratamento inclui a redução de peso } \\
\text { estavam mais controlados ( } \mathrm{p}<0,05 \text {, } \\
24 \% \text { vs } 9 \% \text { ). Assim, o conhecimento } \\
\text { sobre o estilo de vida pode influenciar o } \\
\text { controle de pessoas hipertensas. }\end{array}$ \\
\hline
\end{tabular}


Destaca-se quanto ao período de publicação, do filtro realizado, as primeiras publicações selecionadas que foram do ano de 2010, assim como o maior quantitativo de publicação foi realizado nesse mesmo ano (A2, A3, A8 e A10). A abordagem do tipo de estudos descritivos quantitativos foram destaques entre as publicações (A3, A4, A6, A7, A8, A9 e A10).

\section{A família como sujeito/objeto do cuidado de enfermagem}

O envolvimento familiar no cuidado é maior quando a doença é acompanhada de dependência física. Nesses casos, observa-se que a família, além das atividades voltadas ao portador de HAS com dependência, investe no estímulo a ações de autocuidado e à busca de independência.

A família é, portanto, a base de suporte no controle da doença e é a instituição mais presente em cada indivíduo no apoio às suas necessidades básicas. Na população em estudo, algumas relações são de dependência, seja por cuidadores com vínculo familiar ou não. Muitos recebem o apoio de cuidadores no controle de sua doença.

Ao participar ativamente do cuidado, o familiar cuidador desempenha tarefas importantes que auxiliam no controle dos fatores de risco de hipertensão, além de poder suprir as demandas do tratamento da doença. ${ }^{7}$

Conforme o Artigo $01,{ }^{8}$ os cuidados para uma pessoa idosa devem visar à manutenção de seu estado de saúde, com uma expectativa de vida ativa máxima possível, junto aos seus familiares e à comunidade, com independência funcional e autonomia máximas possíveis. ${ }^{8}$

Portanto, fica evidente que o cuidado com a saúde é um processo que vai além de ensinar, instruir e treinar as pessoas, em especial aquelas com HAS, para mudanças de hábitos de vida. Pressupõe assumir postura política e ética, fundada em valores de solidariedade e justiça, compreendendo o ser humano em essência como ser destinado à liberdade, felicidade e plena realização. ${ }^{9}$

O Artigo $04^{4}$ demonstra que as alterações mais comuns incorporadas pelos familiares incluem a adaptação e participação na realização de atividade como cuidar do tratamento medicamentoso do familiar, adaptar a rotina alimentar e de atividades físicas e a ela aderir, e acompanhar o doente nas consultas médicas. ${ }^{10,11}$

Nessa perspectiva, o processo do cuidar que está inserido na prática profissional do enfermeiro faz interface com todos os membros da equipe de saúde, com a família, com a comunidade e também com o ambiente onde ele executa seu trabalho. Ele está obrigatoriamente introduzido numa relação direta, processual, dialógica, interativa e subjetiva, inerente ao cuidado da vida humana. ${ }^{11,12}$

Os resultados apontados no Artigo $05^{13}$ foram a adesão do cliente ao tratamento integral, o comparecimento às consultas, o uso regular do esquema terapêutico, a adoção de estilo de vida saudável, sobretudo, o compromisso deste com a própria saúde, atuando como sujeito da ação, e não como objeto. Enfim, a adesão do cliente é resultante do seu engajamento efetivo nas atividades de autocuidado, tornando-se agente de autocuidado e multiplicador dessas atividades na família e na comunidade. ${ }^{8}$

Nessa perspectiva, em uma relação entre terapêutica, pacientes e familiares devem ser respeitados, em sua individualidade, direitos e valores. $\mathrm{O}$ paciente precisa ser reconhecido como integrante de uma família; 
por isso, algumas considerações e cuidados devem ser centrados na família, propiciando um clima acolhedor e de proximidade. ${ }^{9,10}$ Essa interação necessita envolver a equipe de enfermagem, o paciente e a família, considerando os aspectos físicos, emocionais, éticos, espirituais e sociais do cuidar. ${ }^{13}$

Dessa maneira, a baixa adesão é mais frequente no nível primário de atenção do que entre pacientes de serviço especializado, sendo importante a distinção entre a pressão não controlada pela não adesão ao tratamento e a resistência ao controle da pressão arterial. Isso porque, a pressão não controlada devido a não adesão ao tratamento é um fator que está relacionado principalmente à conduta do paciente, bem como, ao profissional de saúde e ao Estado. E diferente disso, está a resistência do organismo a determinados fármacos anti-hipertensivos. ${ }^{14,15}$

\section{Reflexos do acolhimento no cuidado dos profissionais de saúde na atenção ao paciente com hipertensão}

É reconhecida a importância da equipe multiprofissional no cuidado à saúde dos hipertensos, pois a mesma pode influenciar positivamente na adaptação da doença e na efetivação da farmacoterapia. $\mathrm{Na}$ equipe, há múltiplos objetivos e abordagens com ação diferenciada, corrigindo a grande limitação no tratamento do hipertensivo, melhorando a adesão ao programa de atendimento e o controle da doença. ${ }^{16}$

A palavra acolhimento traz em si a busca por um atendimento mais humanizado, em que há encontros de subjetividades, tornando a intervenção do cuidado mais efetiva. Vale salientar que acolhimento não deve ser confundido com recepção, pronto atendimento apenas, mas sim com um ato de receber a pessoa a partir de uma escuta qualificada, que permita evidenciar a complexidade da demanda trazida. ${ }^{17}$

As ações em equipe são de fundamental importância na obtenção da motivação dos pacientes, a fim de que estes possam tomar medidas efetivas e permanentes para a prevenção e o controle da HAS. ${ }^{2}$

Nesse sentido, o Artigo $06^{18}$ relata o cuidar como função inerente à enfermagem e, nesse caso, o cuidado ao indivíduo hipertenso, é um fator de extrema relevância, que visa contribuir para o controle e o retardo das complicações da doença. ${ }^{18}$

As reuniões da equipe que atende ao hipertenso devem ser realizadas periodicamente com todos os profissionais envolvidos para a análise crítica das atividades realizadas, acertos de arestas e novas orientações quando houver necessidade. ${ }^{1,19}$

É necessário que os profissionais de saúde tenham um conhecimento sempre atualizado dos aspectos epidemiológicos, diagnósticos e terapêuticos da HAS, a fim de realizar uma correta abordagem dessa entidade clínica (Artigo 07). ${ }^{12,20}$

Os membros de um grupo multiprofissional, respeitada a especificidade de sua formação básica, devem conhecer a ação individual de cada um dos outros membros e, além disso, cada local de trabalho deve se adequar à sua realidade. ${ }^{21,22} \quad \mathrm{O}$ Artigo 06 ainda esclarece que a comunicação é um dos aspectos mais importantes do cuidado de enfermagem que visa uma assistência de qualidade.$^{18} \mathrm{O}$ relacionamento da equipe de enfermagem nesse processo de comunicação 
deve ser eficiente e permanente, viabilizando uma assistência humanista e personalizada. ${ }^{12}$

A experiência tem demonstrado que a atuação de equipes multiprofissionais é facilitadora da educação em saúde e, como consequência, da melhor adesão, por ser composta de indivíduos com formações diferentes, que proporcionam, na ação educativa, maior gama de informações com diversas visões do problema, e esse fato contribui para facilitar o aprendizado. ${ }^{3,20,22}$

Ou seja, cabe aos profissionais de saúde esclarecer não só o tratamento, mas, de acordo com o nível instrucional de cada paciente, o significado da doença e suas implicações fisiopatológicas. ${ }^{20-22}$ Desse modo, os profissionais de saúde precisam ser, antes de tudo, educadores e facilitadores das transformações nas mentes humanas. Para isso, é necessário haver uma boa comunicação/relação entre o profissional e o cliente, e a valorização deste como agente do próprio cuidado (Artigos 07 e 08)..$^{12,20,23}$

Com a multiplicidade de objetivos para o tratamento, são exigidas diferentes abordagens, sendo necessária a formação de uma equipe multiprofissional. ${ }^{23}$ Essa abordagem multiprofissional é particularmente útil no atendimento ambulatorial, ampliando o sucesso do tratamento anti-hipertensivo e do controle dos demais fatores de risco cardiovascular. ${ }^{16}$

\section{Importância da adesão do trata- mento na qualidade dos cuida- dos da hipertensão arterial}

Garantir a eficácia do tratamento proposto tem sido um grande desafio para equipes de saúde. A falta de adesão e consequente controle inadequado da pressão arterial têm sido frequentes e estão associados a diversos fatores. As dificuldades de adesão ao tratamento medicamentoso estão relacionadas especialmente aos efeitos das drogas e custo de medicação. Quanto ao tratamento não farmacológico, observa-se, com frequência, a falta de constância e persistência do paciente. ${ }^{8}$

Referente à adesão ao tratamento, os Artigos 03, 04 e 09 evidenciam que, ao falar de adesão, deve-se sempre considerar a subjetividade que faz que cada indivíduo, de acordo com as suas vivências, conhecimentos, crenças e valores, tenha um comportamento muito próprio em relação ao significado de "sentir-se doente". ${ }^{4,10,24}$ Isso reflete na forma como esse indivíduo se manifesta quando abordamos essas questões. ${ }^{21}$

O controle da pressão arterial e a adesão ao tratamento estão intimamente relacionados, os fatores que interferem na adesão nem sempre são fáceis de detectar e para melhorar o controle da HAS é importante identificar aqueles pacientes que não aderem ao tratamento. ${ }^{9,24}$

Adesão é um processo complexo e um desafio que envolve a atuação tanto dos pacientes como dos profissionais de saúde. Os fatores que interferem na adesão ao tratamento anti-hipertensivo podem ser relacionados ao paciente, como: sexo, idade, raça, escolaridade, etnia, estado civil, nível socioeconômico e religião. Outros fatores estão relacionados com a própria doença, como: cronicidade, conhecimento da patologia e complicações; além destes, há aspectos culturais, crenças, perspectiva dos tratamentos e envolvimento familiar, que devem ser avaliados. O modo como a equipe de saúde se relaciona com os pacientes, o vínculo estabelecido e a especificidade do 
tratamento também influenciam no processo de adesão. ${ }^{10,25-28}$

Entre os critérios de adesão, observou-se no Artigo $02^{9}$ que a dieta apresenta a maior dificuldade para seguimento, representando o menor percentual. Essa realidade pode estar associada ao entendimento de que as restrições parecem castigo por estarem ligadas ao prazer em degustar tais alimentos, ou ainda, associada ao fator econômico que cerceia a liberdade em escolher os alimentos mais adequados. ${ }^{9}$

A adesão terapêutica significa relação colaborativa entre o paciente e os profissionais de saúde, podendo ser caracterizada pelo grau de coincidência entre a prescrição médica e o comportamento do paciente. ${ }^{26}$

Adesão do paciente a determinada terapia depende de vários fatores que incluem, entre outros, os relativos à relação médico-paciente, às questões subjetivas do paciente, às questões referentes ao tratamento, à doença, ao acesso ao serviço de saúde, à obtenção do medicamento prescrito e à continuidade do tratamento. ${ }^{10,27}$

Nesse sentido, a renda familiar e a condição socioeconômica são descritas como fatores associados negativamente à adesão ao tratamento de doenças crônicas. ${ }^{29}$

Alguns fatores dificultam o controle de pressão arterial, como: o desconhecimento da condição de hipertensão; a não adesão do paciente ao tratamento; a não modificação no estilo de vida; a não utilização e/ ou inadequação dos fármacos; a terapêutica inadequada, incapaz de promover o controle da pressão arterial e os gastos econômicos relacionados ao tratamento. ${ }^{2,9,23,30}$

De acordo com o Artigo 05,,$^{13}$ mesmo com a difusão de informações, muitas pes- soas ainda desconhecem os agravos desenvolvidos, não aderem ao tratamento ou o realizam de maneira diferenciada da prescrita nos protocolos.

A respeito de todo conhecimento atualmente acumulado, o percentual de pacientes hipertensos que conhecem a sua condição e tentam controlá-la ainda é muito baixo. Diversas razões podem implicar na baixa taxa de controle de pressão arterial, entre as principais, estão o indivíduo que desconhece a sua condição de hipertenso, a não modificação de estilo de vida, a utilização inadequada dos fármacos e a terapêutica inadequada. ${ }^{12,23,25}$

\section{CONSIDERAÇõES FINAIS}

Identificamos, neste estudo, que são responsáveis pelo absenteísmo ao tratamento da HAS, fatores que dificultam o seu controle e que estão relacionados, principalmente à falta de compreensão e entendimento sobre o assunto, o tratamento, o controle e as complicações. Esses fatores são inerentes ao próprio portador, referindo-se à sua maneira de ser e de agir.

Ao falarmos sobre adesão ao tratamento, devemos sempre considerar a subjetividade que faz que cada indivíduo, de acordo com as suas vivências, conhecimentos, crenças e valores, tenha um comportamento muito próprio em relação ao significado de "sentir-se doente". Isso reflete na forma como esse indivíduo se manifesta quando abordamos essas questões. Dessa maneira, também não podemos desconsiderar as crenças da equipe de saúde, as quais, muitas vezes, podem não coincidir com as crenças e os interesses do paciente. 
É imprescindível identificar as dificuldades com vistas a uma melhor adesão ao tratamento da hipertensão, bem como, das doenças crônicas associadas, reforçando a necessidade de se informar e discutir as condições observadas pelos membros da equipe de saúde.

Evidenciamos que, apesar de muitos pacientes mencionarem atitudes positivas em relação ao tratamento medicamentoso e demonstrarem bom nível de conhecimento sobre a doença e o tratamento, o controle da pressão arterial ainda não é satisfatório, caracterizando a necessidade de medidas que visem melhor o seu controle, tornando o tratamento um problema que deve ser enfrentado por todos: hipertenso, família, comunidade, instituições e equipe de saúde.

Espera-se, com este estudo, contribuir de alguma maneira para que os profissionais de saúde que lidam com pessoas portadoras de HAS consigam esclarecê-las, devidamente, sobre sua doença e tratamento, no intuito de diminuir o índice de alterações clínicas que ocorrem em decorrência da HAS.

Além dos pacientes, cuidadores, familiares e membros da equipe de saúde, devem se sentir incluídos nesse processo de tratamento da hipertensão e da resolução dos problemas e dificuldades encontrados nesse sentido, pesquisadores, governo e sociedades médicas e de áreas afins, com o objetivo de aumentar a adesão ao tratamento e possibilitar um melhor controle da hipertensão.

\section{REFERÊNCIAS}

1. MINISTÉRIO DA SAÚDE. Brasil. Linhas de cuidado: hipertensão arterial e diabetes. / Organização Pan-Americana da Saúde. Brasília: Organização Pan-Americana da Saúde, 2010.

2. Sociedade Brasileira de Cardiologia. VI Diretrizes Brasileiras de Hipertensão. Rev Bras Cardiol., 2010; 17: 25-30.

3. OLIVEIRA TL, MIRANDA LP, FERNANDES PS, CALDEIRA AP. Eficácia da educação em saúde no tratamento não medicamentoso da hipertensão arterial. Acta Paul Enferm. [online]. 2013; 26 (2): 179-184.

4. RIBEIRO IJS, BOERY RNSO, CASOTTI CA, FREIRE IV, TEIXEIRA JRB, BOERY EM. Prevalência e fatores associados à adesão ao tratamento medicamentoso por pacientes com hipertensão arterial. Rev Baiana Enferm. 2015; 29 (3): 250-260.

5. BORGES JWP. Instrumento de avaliação da não adesão ao tratamento da hipertensão arterial: desenvolvimento e validação de conteúdo [dissertação]. Fortaleza (CE): Universidade Estadual do Ceará, 2012.

6. SOARES CB, HOGA LAK, PEDUZZI M., SANGALETI C., YONEKURA T., SILVA DRAD. Integrative review: concepts and methods used in nursing. Rev Esc Enferm USP, 2014; 48 (2): 335-45. Doi: http://dx.doi.org/10.1590/S0080-6234201400002000020

7. COHEN MJ, SHAYKEVICH S., CAWTHON C., KRIPALANI S., PAASCHE-ORLOW MK, SCHNIPPER JL. Predictors of medication adherence postdischarge: the impact of patient age, insurance status, and prior adherence. J Hosp Med., 2012; 7 (6): 470-5.

8. BEZERRA ASM, LOPES JL, BARROS ALBL. Adesão de pacientes hipertensos ao tratamento medicamentoso. Rev Bras Enferm., 2014; 67 (4): 550-5.

9. FIGUEIREDO NN, ASAKURA L. Adesão ao tratamento anti-hipertensivo: dificuldades relatadas por indivíduos hipertensos. Acta Paul Enferm., 2010; 23 (6): 782-7. 
10. DUARTE MTC, CYRINO AP, CERQUEIRA ATAR, NEMES MIB, IYDA M. Motivos do abandono do seguimento médico no cuidado a portadores de hipertensão arterial: a perspectiva do sujeito. Ciênc Saúde Colet., 2010; 15 (5): 2.603-2.610.

11. GUSMAO JL, MION JUNIOR D. Adesão ao tratamento: conceitos. Rev Bras Hipertens. 2006; $13(1): 23-5$.

12. MENDES LMO, BARROS JST, BATISTA NNLAL, SILVA JMO. Fatores associados a não adesão ao tratamento da hipertensão arterial sistêmica: uma revisão integrativa. Rev Univap., 2014; 20 (35).

13. ALMEIDA GBS, PAZ EPA, SILVA GA. Representações sociais de portadores de hipertensão arterial sobre a doença: o discurso do sujeito coletivo. Rev Min Enferm., 2013; 17 (1): 46-53.

14. SIQUEIRA BPJ, TEIXEIRA JRB, VALENÇA NETO PF, BOERY EM, BOERY RNSO, VILELA ABA. Men and health care in the social representations of health professionals. Esc Anna Nery., 2014; 18 (4): 690-696.

15. CARVALHO FILHA FSS, NOGUEIRA LT, MEDINA MG. Avaliação do controle de hipertensão e diabetes na Atenção Básica: perspectiva de profissionais e usuários. Saúde Debate., 2014; 38: 265-278.

16. MATTHES J, ALBUS C. Improving adherence with medication: a selective literature review based on the example of hypertension treatment. Dtsch Arztebl Int. Cologne., 2014, 111 (4): 41-47.

17. AYRES JRCM. Cuidado: Trabalho e Interação nas Práticas de Saúde (Coleção Clássicos para Integralidade em Saúde). Rio de Janeiro: Centro de Estudos e Pesquisa em Saúde Coletiva, Instituto de Medicina Social, Universidade do Estado do Rio de Janeiro/ABRASCO, 2011.

18. MEDEIROS ARC, ARAÚJO YB, VIANNA, RPT, MORAES RM. Modelo de suporte à decisão aplicado à identificação de indivíduos não aderentes ao tratamento anti-hipertensivo. Saúde Debate, 2014; 38 (100): 104-118.

19. LIMA TM, MEINERS MMMA, SOLER O. Perfil de adesão ao tratamento de pacientes hipertensos atendidos na Unidade Municipal de Saúde de Fátima, em Belém, Pará, Amazônia, Brasil. Rev Pan Amaz Saúde, 2010; 1 (2): 113-120.

20. MACHADO JC, COTTA RMM, MOREIRA TR, SILVA LS. Análise de três estratégias de educação em saúde para portadores de hipertensão arterial. Ciênc Saúde Colet, 2016; 21 (2): 611-620.

21. FAVA SMCL, TERAOKA EC, OLIVEIRA AS, CALIXTO AATF, EID LP, VEIGA EV. Fatores relacionados à adesão ao tratamento da hipertensão arterial sistêmica. Rev Rene., 2014; 15 (2): 354-61.

22. FELIPE GF, MOREIRA TMM, SILVA LF, OLIVEIRA ASS. Consulta de enfermagem ao usuário hipertenso acompanhado na atenção básica. Rev Rene., 2011; 12 (2): 287-94.

23. UNGARI AQ, DAL FABBRO AL. Adherence to drug treatment in hypertensive patients on the Family Health Program. Braz J Pharm Sci., 2010; 46 (4): 811-18.

24. SANTOS ZMSA, LIMA HP, OLIVEIRA FB, VIEIRA JS, FROTA NM, NASCIMENTO JC. User's adherence to hypertensive drug therapy. Rev Rene., 2013; 14 (1): 11-22.

25. SERAFIM TS, JESUS ES, PIERIN AMG. Influência do conhecimento sobre o estilo de vida saudável no controle de pessoas hipertensas. Acta Paul Enferm., 2010; 23 (5): 658-664.

26. TRIVEDI RB, AYOTTE B, EDELMAN D, BOSWORTH HB. The association of emotional wellbeing and marital status with treatment adherence among patients with hypertension. J Behav Med., 2008; 31 (6): 489-97.

27. SANTA HELENA ET, NEMES MIB, ELUF NETO J. Fatores associados à não-adesão ao tratamento com anti-hipertensivos em pessoas atendidas em unidades de saúde da família. Cad Saúde Publ., 2010; 26 (12): 2.389-98. 
28. DEMONER MS, RAMOS ERP, PEREIRA ER. Fatores associados à adesão ao tratamento anti-hipertensivo em unidade básica de saúde. Acta Paul Enferm., 2012; 25 1): 27-34.

29. WORLD HEALTH ORGANIZATION. Adherence to long-term therapies: evidence for action. Geneva: WHO; 2003. Disponível em: http://www.who.int/chp/knowledge/publications/adherence_full_report.pdf.

30. KIM JH, LEE WY, HONG YP, RYU WS, LEE KJ, LEE WS et al. Psychometric properties of a Short Self-Reported Measure of Medication Adherence among patients with hypertension treated in a busy clinical setting in Korea. J Epidemiol., 2014; 24 (2): 132-140.

\section{DAdos DOS AUTORES}

\section{Silas Santos Carvalho}

Mestre em Saúde Coletiva pela Universidade Estadual de Feira de Santana-BA (UEFS). Enfermeiro com Pós-graduação em Enfermagem Obstétrica e Saúde da Mulher pela Faculdade Anísio Teixeira (FAT) de Feira de Santana. Integrante e pesquisador do Núcleo de Pesquisa, Prática Integrada e Investigação Multidisciplinar - NUPPIIM (UEFS). Feira de Santana/BA - Brasil. ssc.academico@ hotmail.com

\section{Bruno Rodrigues de Oliveira}

Administrador. Analista de Planejamento. Universidade de Salvador. Feira de Santana/BA - Brasil. brodrigues.tel@gmail.com

Submetido em: 28-2-2018

Aceito em: 5-11-2019 